\title{
Straight Back Syndrome with Heart Failure and Myocardial Injury Diagnosis by Cardiac Magnetic Resonance
}

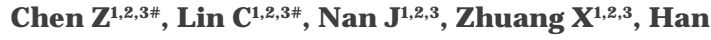 \\ $\mathrm{B}^{4}$, Lei J $\mathrm{J}^{1,2,3 *}$, Guo $\mathrm{S}^{1,2,3 *}$, Xue J ${ }^{1,2,3}$, Zhang $\mathrm{Z}^{4}$, Lu $\mathrm{M}^{5}$ \\ and Zhao $S^{5}$ \\ 'Department of Radiology, The First Hospital of Lanzhou \\ University, Lanzhou, 730000, People's Republic of China \\ ${ }^{2}$ Intelligent Imaging Medical Engineering Research \\ Center of Gansu Province, China \\ ${ }^{3}$ Accurate Image Collaborative Innovation International \\ Science and Technology, Cooperation Base of Gansu \\ Province, Lanzhou, China \\ ${ }^{4}$ Department of Cardiology, The First Hospital of Lanzhou \\ University, Lanzhou, People's Republic of China \\ ${ }^{5}$ Department of Magnetic Resonance Imaging, \\ Cardiovascular Imaging and Intervention Center, \\ State Key Laboratory of Cardiovascular Disease, Fuwai \\ Hospital, National Center for Cardiovascular Diseases, \\ Chinese Academy of Medical Sciences and Peking Union \\ Medical College, Beijing, People's Republic of China \\ ${ }^{\#}$ Contributed Equally to this work \\ *Corresponding author: J unqiang Lei, Department of \\ Radiology, The First Hospital of Lanzhou University, No. \\ 1, Donggang West Road, Chengguan District, Lanzhou \\ City, Gansu Province, 730000, People’s Republic of China \\ Shunlin Guo, Department of Radiology, The First \\ Hospital of Lanzhou University, No. 1, Donggang West \\ Road, Chengguan District, Lanzhou City, Gansu Province, \\ 730000, People's Republic of China
}

Received: April 29, 2021; Accepted: May 17, 2021;

Published: May 24, 2021

\section{Introduction}

Straight Back Syndrome (SBS) is an autosomal dominant hereditary disease characterized by abnormal thoracic skeletal dysplasia, which leads to a decrease in anteroposterior diameter of the thorax and thereby the heart and great vessels are compressed and displaced [1]. Patients are usually asymptomatic and cardiac morphology and functions are normal. It typically occurs in young women [2]. We report a middle-aged man who was eventually diagnosed as SBS with secondary heart failure and myocardial injury by Cardiac Magnetic Resonance (CMR).

\section{Case Presentation}

A 46-year-old Chinese man was referred to our hospital for intermittent heart palpitations, chest tightness for four months. The Holter in the local hospital showed frequent ventricular premature and short paroxysmal ventricular tachycardia. The Transthoracic Echocardiography (TTE) demonstrated Left Ventricular (LV) enlargement and $55 \%$ of ejection fraction. Coronary angiography was normal. Then he was diagnosed as dilated cardiomyopathy and given symptomatic treatment with drugs (Noxinto, metoprolol sustainedrelease tablets, spironolactone). After a slight relief of symptoms, he was advised to undergo ICD treatment. The patient was admitted to our hospital for further definitive diagnosis.

The patient had no previous medical history or family history except for heart murmurs found during routine examination at the age of 18. Physical examination showed blood pressure was 116/69 $\mathrm{mmHg}$ and heart rate was 78 beats/min. His chest was flat. Cardiac auscultation revealed Grade 1/3 systolic ejection murmur with fixed splitting of the second heart sound, auscultated between the 2 to 3 ribs of the left sternal border. Grade $2 / 6$ systolic murmurs were auscultated at the mitral area (Figure 1).

Laboratory evaluation showed that NT-proBNP was $482 \mathrm{pg} / \mathrm{ml}$, homocysteine was elevated $(20.14 \mu \mathrm{mol} / \mathrm{L})$ and other serum cardiac biomarkers were negative. The electrocardiograph was abnormal (Figure 2A and 2B) and revealed QS pattern in leads V1-V2. Holter demonstrated frequent ventricular premature beats (right ventricular outflow tract origin) with short ventricular tachycardia and occasional atrial premature beats. Frontal chest radiography showed mild
Austin J Cardiovasc Dis Atherosclerosis - Volume 8 Issue 1 - 2021 ISSN: 2472-3568 | www. austinpublishinggroup.com

Chen et al. $\odot$ All rights are reserved
Citation: Chen Z, Lin C, Nan J, Zhuang X, Han B, Lei J, et al. Straight Back Syndrome with Heart Failure and Myocardial Injury Diagnosis by Cardiac Magnetic Resonance. Austin J Cardiovasc Dis Atherosclerosis. 2021; 8(1): 1043. 


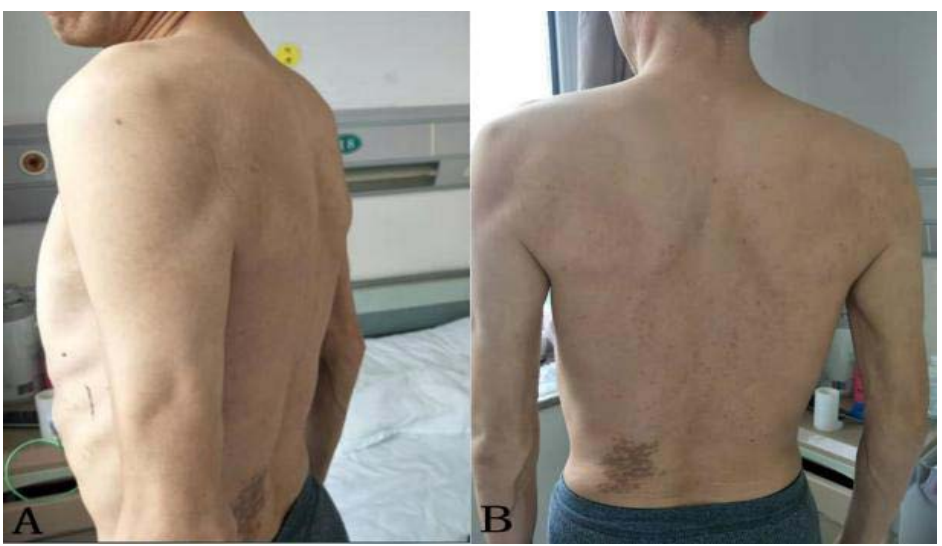

Figure 1: Lateral view (A) and posterior view (B) show the narrow chest and the straight thoracic spine.
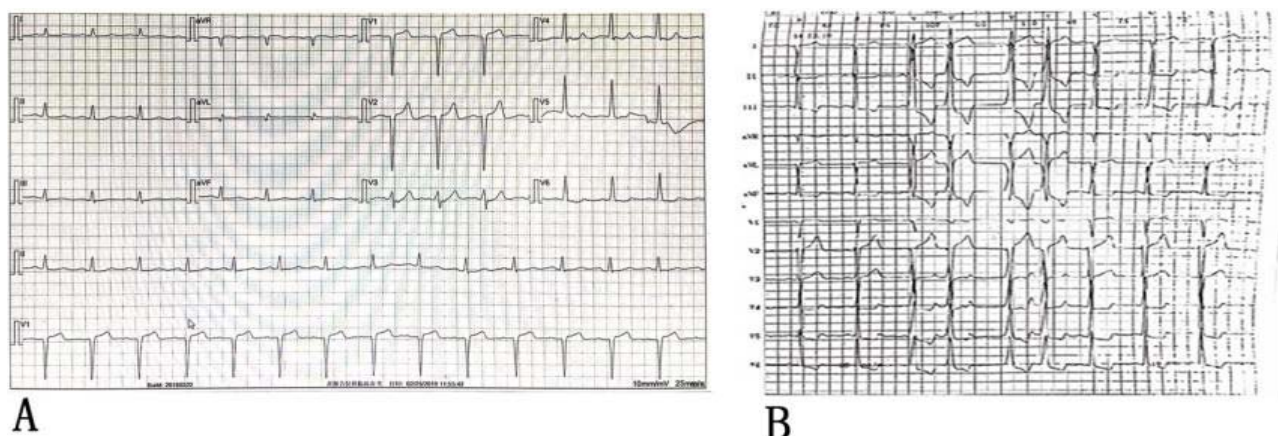
B

Figure 2: Electrocardiogram shows QS pattern in leads $\mathrm{V} 1-\mathrm{V} 2$ (a). Ventricular premature beat origin from right ventricular outflow tract (b).

pulmonary artery distension and LV enlargement. TTE demonstrated left atrial and LV enlargement with LV dysfuction (EF43\%), mitral and tricuspid valve regurgitation with anterior Mitral Valve Prolapse (MVP). None of these diagnoses were specific, then we performed CMR using a 3T scanner (Skyra, Siemens Healthcare, Forchheim, Germany) for further clarify the diagnosis.

CMR black blood images showed a markedly shortened anteroposterior diameter of the chest. The ratio of anteroposteriorto-transverse diameter was 0.29 which achieved the diagnostic criteria for straight back syndrome. Multiplanar cine ( $3 \mathrm{ch}, 4 \mathrm{ch}$, sax) images showed the LV enlargement and global wall motion weakened. CMR confirmed the presence of mitral valve regurgitation due to anterior mitral valve prolapse and the anterior mitral valve leaflet elongation $(35 \mathrm{~mm})$. Intravoxel Incoherent Motion (IVIM) sequence and Late gadolinium enhancement (LGE) images showed hypoperfusion of ventricular septum and anterior wall in left ventricle and myocardial fibrosis in ventricular septum (Figure 3).

\section{Discussion}

Straight Back Syndrome (SBS) was an autosomal dominant hereditary disease, which characterized by absence of the normal upper thoracic kyphosis, leading to a reduced anteroposterior diameter of the thorax [1]. The most patients are asymptomatic, but some of them may present with chest pain, palpitation and dyspnea with heart murmur and arrhythmia, because of the heart and great vessels compression $[3,4]$.

Arrhythmia was usually manifested as sinus tachycardia, ventricular premature beat and bundle branch block [1]. Previous literature reported that the origin of arrhythmia is related to the compression site of the heart [5]. Mechanical-electrical feedback plays an important role in it. This patient has frequent ventricular premature beats which origin from right ventricular outflow tract, consistence with previous literature.

Reports of patients with straight back syndrome with cardiac dysfunction and myocardial injury are extremely rare [6].The main manifestations of our case were heart failure and arrhythmia. CMR demonstrated hypoperfusion of myocardial and myocardial fibrosis. We speculated that the myocardial injury secondary to the long-term mechanical premature beats.

SBS was commonly associated with MVP and the proportion of SBS combined with MVP was as high as $67 \%[3,7]$. Both skeleton and heart valves belong to mesodermal development, which was completed around the eighth week of embryonic development. Any factors that affect growth at this stage may affect both systems. Hence, some authors proposed that they may have a common pathogenic gene [3]. In addition to MVP, our patient also demonstrated the anterior mitral valve leaflet elongation. Whether long anterior mitral leaflet is also one of the characteristics of SBS needs further large sample study and gene confirmation. 
A

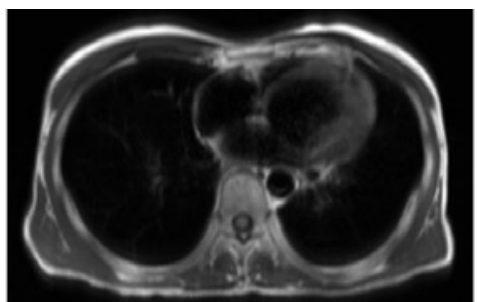

B

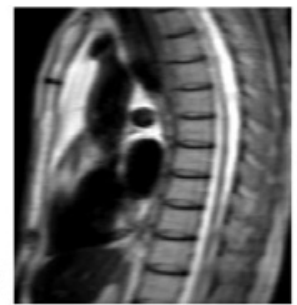

D

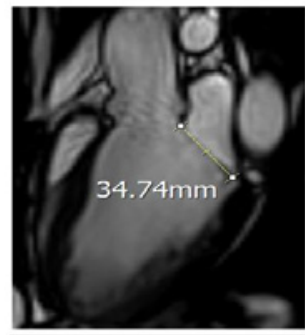

G

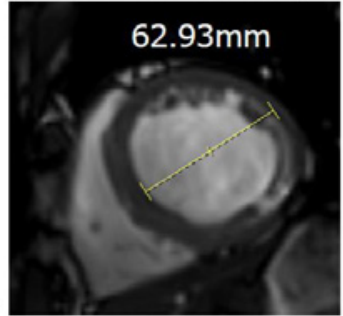

E
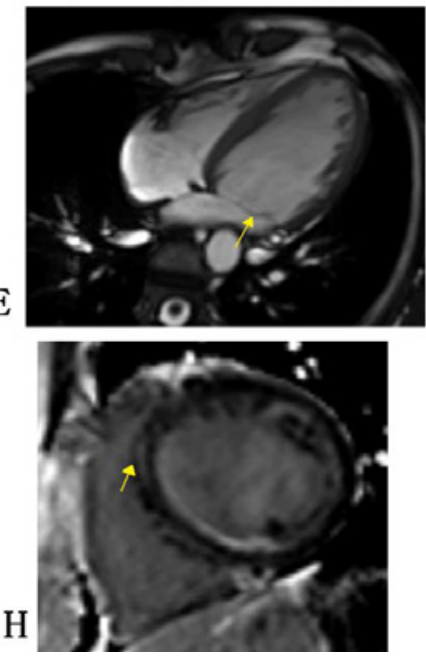

C

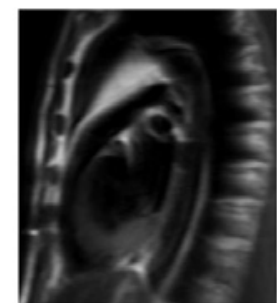

$\mathrm{F}$
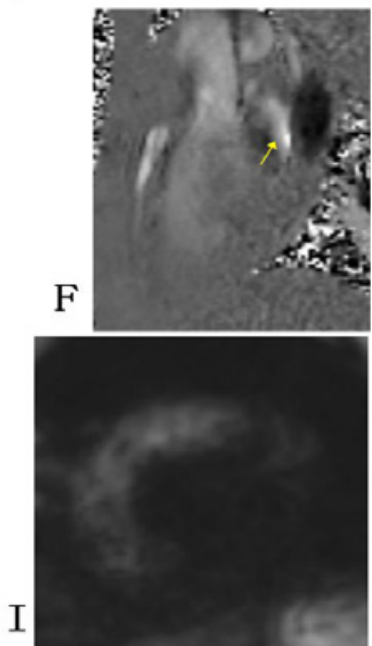

Figure 3: Patinet's CMR images. A-C: Haste black blood images shows a reduction in anteroposterior diameter of the thorax, right ventricular, right ventricular outflow tract, left atrial compression. D, E: Three-chamber and four-chamber cine imaging shows anterior mitral elongation and MVP (arrow). F: Three-chamber flow imaging shows mitral regurgitation (arrow). G: Left ventricular mid-axis short-axis cine imaging shows LV end-diastolic diameter increased. H: LGE imaging shows ventricular septal myocardial fibrosis (arrow). I: IVIM imaging $(b=20)$ shows septal and anterior myocardial signal increase.

Finally, the patient was diagnosed with SBS instead of dilated cardiomyopathy.

\section{Conclusion}

We reported a case with SBS showing cardiac dysfunction and myocardial injury by CMR. This case should remind physicians of the importance of differential diagnosis in patients with heart failure and arrhythmia. SBS may need to be considered in patients without organic heart disease.

\section{Acknowledgement}

This work was also partially supported by the Department of Magnetic Resonance Imaging, Cardiovascular Imaging and Intervention Center, State Key Laboratory of Cardiovascular Disease, Fuwai Hospital, National Center for Cardiovascular Diseases, Chinese Academy of Medical Sciences and Peking Union Medical College.

\section{Grant Support}

This study was supported, in parts, by the Gansu Province Youth Science and Technology Foundation (18JR3RA364) and The First Hospital of Lanzhou University Hospital Foundation (ldyyyn2015-06).

\section{References}

1. Rawlings MS. The "straight back" syndrome, a new cause of pseudoheart disease. Am J Cardiol. 1960; 5: 333-338.

2. Esser SM, Monroe MH, Littmann L. Straight back syndrome. Eur Heart J. 2009; 30: 1752.

3. Davies MK, Mackintosh P, Cayton RM, Page AJ, Shiu MF, Littler WA. The straight back syndrome. Q J Med. 1980; 49: 443-460.

4. Grillo HC, Wright CD, Dartevelle PG, Wain JC, Murakami S. Tracheal compression caused by straight back syndrome, chest wall deformity, and anterior spinal displacement: techniques for relief. Ann Thorac Surg. 2005; 80: 2057-2062.

5. Chen $\mathrm{L}, \mathrm{Ma} X \mathrm{XH}$, Zhao $\mathrm{L}$, et al. [A preliminary study on the relationship between idiopathic arrhythmia and cardiac magnetic resonance imaging defined cardiac features in patients with straight back syndrome]. Zhonghua Xin Xue Guan Bing Za Zhi. 2017; 45: 948-953.

6. Ryo-Koriyama K, Matsumoto K, Nishii T, Tanaka H, Hirata K. A rare case of double-chambered right ventricle apparent on the compression by both pectus excavatum and straight back syndrome. Eur Heart J Cardiovasc Imaging. 2016; 17: 706.

7. Ansari A. The "straight back" syndrome: current perspective more often associated with valvular heart disease than pseudoheart disease: a prospective clinical, electrocardiographic, roentgenographic, and echocardiographic study of 50 patients. Clin Cardiol. 1985; 8: 290-305. 